\title{
El cambio en el proceso editorial de la revista Neurocirugía
}

\author{
R.D. Lobato; P. González; P.A. Gómez; R. Alday; A. Lagares y J.F. Alen
}

Servicio de Neurocirugía. Hospital 12 de Octubre. Madrid.

\section{Resumen}

En breve se va a introducir un cambio en el proceso editorial de la revista Neurocirugía, órgano oficial de expresión de la Sociedad Española de Neurocirugía (SENEC). Con tal motivo se hace una breve semblanza del recorrido de la revista desde su aparición en 1991 hasta el momento actual. Se consideran algunos cambios ocurridos en los últimos años en el proceso global de la publicación en biomedicina, y más concretamente en la manera de circular los artículos, la metodología de la revisión por pares, y la edición en medios electrónicos, destacando la necesidad de reducir en parte la edición en papel en favor de la electrónica. Se contemplan también otros aspectos relacionados con Neurocirugía, como son la pertinencia y utilidad de disponer de una revista especializada de ámbito nacional, el problema de la lengua a elegir para la presentación de los originales, y la importancia de que los neurocirujanos trasladen a la revista no sólo sus observaciones científicas, sino también opiniones y reflexiones sobre el devenir y la historia de nuestra especialidad.

PALABRAS CLAVE: Neurocirugía. Revista neuroquirúrgica. Publicación. Ciencia biomédica. Revisión por pares. Bibliometría. Medlin. Institute Scientific Information.

The change in the editorial process of Neurocirugia, the journal of the Spanish Neurosurgical Society

\section{Abstract}

The editorial process of Neurocirugia, which is the official journal of the Spanish Neurosurgical Society, will be changed in the immediate future. Together with the announcement of new instructions to the authors, we analyze some recent trends in the process of publication of biomedical research related to peer review methodology and the controversy of the "authors pays"

Recibido: 10-04-06 vs "reader pays" models. The reasons for sustaining a national neurosurgical journal, the advantages for using either Spanish or English to writing the manuscripts, and the importance for Spanish neurosurgeons to reflect some of their scientific contributions and opinions concerning the present and future of our speciality in Neurocirugia are commented.

KEY WORDS: Neurosurgery. Neurosurgical journal. Publication. Biomedical sciences. Peer review. Bibliometry. Medline. Institute Scientific Information.

\section{Introducción}

Este artículo, que aparece junto con las editoriales escritas por Juan Sauquillo, Javier Ibáñez y Máximo Poza, Editor, con motivo de la puesta en marcha de un nuevo método para circular y evaluar los artículos remitidos para valoración, incluye una breve semblanza de la andadura de la revista desde su aparición, un comentario sobre la necesidad de adaptar el nuevo proceso editorial a las recientes tendencias en el mundo de la publicación biomédica, y algunas consideraciones sobre la conveniencia de contribuir a la revista, aunque sea sólo como ejercicio autoeducativo; este último apartado está dedicado a los neurocirujanos más jóvenes, que han de ejercitarse en la dura disciplina de escribir. Además, recalcamos la importancia del cuidado de este valioso instrumento que los neurocirujanos españoles nos hemos otorgado, advirtiendo contra cualquier actitud de relajación apoyada en la creencia de que Neurocirugía es un árbol firmemente enraizado que puede crecer por si sólo

Breve historia de Neurocirugía desde su aparición en 1991

Neurocirugía nació en un momento apropiado del devenir de la Sociedad Española de Neurocirugía (SENEC), entonces todavía Sociedad Lusoespañola. En efecto, su aparición una o dos décadas antes hubiera encontrado una agrupación de neurocirujanos todavía escasa en número 
y con una masa crítica inferior a la necesaria para nutrir una publicación periódica, que en principio fue cuatrimestral. En las primeras etapas de la Sociedad Lusoespañola algunos de sus miembros publicaban trabajos de manera errática, que aparecían en forma de libros de resúmenes en la Revista Española de Oto-Neuro-Oftalmología, o de trabajos completos que no eran seleccionados mediante revisión por pares. A principios de la década de los noventa se había implantado en nuestra Sociedad, nutrida ya de un número proporcionalmente mayor de neurocirujanos jóvenes, una mentalidad más abierta y un hábito más democrático; por otra parte, algunos de ellos se habían mostrado capaces de publicar en revistas extranjeras con un alto nivel de exigencia para la aceptación de originales. Parecía así que nuestra Sociedad contaba finalmente con un grupo de neurocirujanos preparados para sostener una publicación periódica propia en la que reflejar sus actividades científicas y docentes, así como otros aspectos relevantes de su vida y ámbito profesionales. En otras palabras, parecía imprescindible disponer de un órgano de expresión oficial, similar a los que ostentaban ya otras Sociedades europeas.

Con la ayuda de un comité editorial numéricamente escaso y casero (compuesto por miembros de nuestra Sociedad) se editó el primer número de Neurocirugía en 1991. La experiencia de los miembros del comité editorial en la evaluación de trabajos era prácticamente nula, ya que casi ninguno formaba parte entonces de comités de otras revistas y algunos ni siquiera habían realizado publicación alguna, por lo que su capacidad para actuar como revisores era muy limitada, e incluso cuestionable. En cualquier caso, se trataba de iniciar el camino y aprender. Sin embargo, el mérito mayor en el lanzamiento y consolidación de Neurocirugía ha sido siempre, pero más particularmente en aquella era arcaica cuando la revista no estaba incluida en Medline, el de los autores de los artículos aparecidos en ella; en efecto, gracias a su oferta la revista tomó cuerpo y pudo sostener el ritmo de su aparición periódica imprescindible para solicitar su aceptación en bases de datos como Research Alert Institute for Scientific Information, CAB, EMBASE/Excerpta Medica y otros repertorios de ámbito internacional.

Una vez consolidada y entronizada en las redes de datos mencionadas, se complementó el comité editorial con algunos colegas extranjeros y surgió el ambicioso proyecto de acceder a Medline/Index Medicus, y quizás también al Institute for Scientific Information, (ISI) aún sabiendo que estos últimos objetivos, meta ideal de cualquier publicación biomédica de aparición periódica, eran difíciles de conseguir. Tras asesorarnos acerca del protocolo y los pasos a seguir para que la revista fuera aceptada en la National Library y su red Medline, dirigimos una solicitud que fue contestada en una amable carta en la que se nos informaba de la necesidad de remitir varios números consecutivos ya publicados para valoración, y superar además un riguroso examen por parte de un comité que se reunía periódicamente. La respuesta inicial fue de rechazo, aun cuando Neurocirugía había puntuado por encima de 3 sobre 5 en cuatro de los cinco ítems de valoración, alcanzando 4 puntos en uno de los ítems y 5 en otro; sin embargo, la carta abría la esperanza de poder superar el filtro en la siguiente convocatoria, y exigía seguir remitiendo prospectivamente los números publicados. En este largo proceso recibimos la ayuda de Javier Ibáñez y Juan Sahuquillo que conocían muy bien las normas del ISI, cuya adopción por la revista aumentaba sus posibilidades de ser finalmente aceptada. Tras una larga espera Neurocirugía fue incluida en la edición del Journal of Citation Reports del año 1998, organismo dependiente del ISI que calcula y difunde el "factor de impacto", y aceptada en Medline/Index Medicus. A raíz de la aceptación apareció el excelente estudio bibliométrico de Ibáñez y cols ${ }^{12}$, que debe ser releído de vez en cuando, al menos por quienes estén implicados en el proceso editorial.

Aunque estos logros puedan representar una satisfacción y un privilegio para los neurocirujanos españoles, debe movernos a reflexión el hecho de que este paso aparentemente trascendental no ha conllevado un incremento significativo ni en el número de originales ofertados por la comunidad neuroquirúrgica española, ni en su calidad media, siendo uno incapaz de aportar una explicación plausible para este hecho paradójico. Tras un recorrido por una planicie en la que Neurocirugía ha mantenido un nivel sin variaciones significativas, la revista recibe un número creciente de artículos de países de América, Europa, y Turquía entre otros, tendencia que debe ser considerada como estimulante; sus autores son neurocirujanos extranjeros de habla española, nativos que envían sus manuscritos en inglés, o bien españoles que realizan allí su especialización y que escriben en cualquiera de estas dos lenguas.

Otro progreso más reciente ha sido la edición de un número extraordinario anual de Neurocirugía con los resúmenes de los trabajos presentados a las reuniones de la SENEC. Ello tiene dos ventajas; por un lado los resúmenes adquieren validez curricular, ya que así pueden ser citados en cualquier revista, y por otro, esta opción conlleva un mejor aprovechamiento de la inversión que antes afrontaba el organizador de la reunión anual para financiar los antiguos libros de resúmenes, cuyos contenidos no se aceptan para ser citados en las revistas indexadas.

Un problema permanente de Neurocirugía, común por lo demás a la mayoría de las publicaciones periódicas, y que hay que reconsiderar en la medida en que se desarrollan más y más las ediciones electrónicas, es el de la financiación de su edición. Es obvio que la edición es responsabilidad de la SENEC, pero también está claro que lo ideal es detraer la mínima cantidad de dinero de sus arcas, ya que la 
repleción depende casi sólo de las cuotas de sus miembros. Gracias a la paciente y cuidadosa gestión de los Editores de la Revista, primero José María Izquierdo, y ahora Máximo Poza, las entradas por publicidad han permitido enjugar la mayor parte del coste de publicación de la revista, dejando así fondos suficientes para afrontar el pago de premios y otros gastos. Sin embargo, es oportuno comentar aquí que las firmas comerciales que hacen las aportaciones por publicidad son las mismas que proporcionan ayudas para la asistencia y organización de los congresos anuales de la SENEC, y quizás sería conveniente que la Junta Directiva de la SENEC interviniera de alguna manera en este asunto incitando al orden y la moderación en nuestras demandas de ayuda.

Según informó el Editor en la Asamblea de la última reunión de Palma de Mallorca, hemos tenido la fortuna de obtener una Beca del Instituto Carlos III creada para mejorar la difusión en el mundo castellano parlante de las 19 revistas españolas que hace cuatro años estaban incluidas en el ISI; para obtener dicha beca era condición necesaria editar al menos una mayoría de artículos en castellano; a cambio se nos ofrecía la entrada y permanencia gratuitas en la red Scielo, cuya entidad cuantitativa es similar a la de Medline; Javier Ibáñez redactó la memoria de solicitud de tal ayuda que nos fue concedida por una cantidad total de 15.000 euros, y que ha permitido paliar el problema de la financiación por un tiempo.

La revista Neurocirugía ante el cambio en el proceso editorial de las revistas biomédicas relacionado con la revisión por pares y la publicación en medios electrónicos.

El proceso global de la publicación de trabajos científicos está sometido a tensiones, algunas de viejo cuño que se reactivan de tiempo en tiempo, y otras de nueva aparición a tenor del desarrollo de las nuevas tecnologías de difusión de la información. Así, se analizan y cuestionan aspectos tales como cuál debe ser el contenido ideal de una revista médica, cuál la estructura de los trabajos, cuál la metodología de circulación de los mismos (presentación en papel, o a través por la red), cómo debe desarrollarse la revisión por pares (abierta, cegada, etc), y cuál debería ser el soporte de la edición (papel vs electrónico) 2,4,5,9,20-23. Todos estos puntos deben ser considerados en relación con el futuro inmediato de Neurocirugía y el cambio que se quiere introducir en el proceso editorial.

Durante un tiempo en el Comité Editorial de Neurocirugía, y al igual que ocurre en todas las revistas, no han estado todos los que son, ni son todos los que están. Así, si bien la revisión de los trabajos ha sido llevada a cabo por la mayoría de sus miembros, de manera lentamente progresiva, y también al igual que se hace en otras revistas, se ha ido requiriendo la ayuda de otros neurocirujanos no incluidos en el mismo. En el último número de Neurocirugía (Febrero,2006) apareció el listado de colegas que no estando incluidos en el Comité Editorial oficial, revisaron trabajos durante el año 2005. Esta participación debe incrementarse por razones de eficacia del propio proceso editorial, y para aprovechar y reconocer mejor la capacidad de muchos de nuestros colegas. Por ello, la oferta hecha por nuestro Editor en la asamblea de Palma de Mallorca para incorporarse a esta labor, se reitera en este momento por escrito. Así, ninguno de los miembros de la SENEC podrá sentirse marginado de esta laboriosa pero estimulante labor, que al ser aceptada incitará también a los nuevos revisores a publicar, contribuyendo así al engrandecimiento de Neurocirugía.

A partir del mes de Septiembre/06 se modificará la circulación y evaluación de los artículos enviados a Neurocirugía, adoptando el sistema de envío por correo electrónico, que durante un tiempo coexistirá con el sistema convencional de envío por correo de superficie. En esencia, un grupo de colegas se encargaran de enviar por correo electrónico los trabajos a un conjunto de revisores que realizarán la evaluación y se comunicarán con ellos, acelerando y facilitando así de manera notable el engorroso proceso editorial que se mantenía hasta ahora. Se espera con ello que los trabajos sean mejor evaluados y que los autores puedan recibir una ayuda mayor y más rápida para mejorar los manuscritos. El anuncio formal de la introducción de este cambio ha sido incluido por el Editor en la hoja de instrucciones a los autores que aparece en el presente número de la revista. En la preparación de este cambio hemos recibido la ayuda técnica inestimable de Segismundo Izquierdo y Pedro González León.

$\mathrm{Al}$ afrontar esta nueva etapa conviene comentar las consideraciones y sugerencias de R. Smith, editor del British Medical Journal, en relación con el desarrollo futuro de la publicación en biomedicina. En una reciente editorial escrita con motivo de su retirada, Smith ${ }^{22}$ comenta la tendencia innata al conservadurismo de los cuerpos editoriales de las revistas médicas, y la necesidad de rediseñarlas para evitar que sean relicarios en los que se ofrecen a los médicos montones de artículos que no leen y que muchas veces no están preparados para valorar. Dice Smith, "Si tuviéramos más claro cual es el propósito de las revistas entonces podríamos rediseñarlas completamente. Poco a poco, el contenido de las revistas pasaría de ser en su mayoría, estudios originales - de los cuales sólo un 1\% aproximadamente resultan tanto válidos como relevantes para los médicos - a ser un material de debate, instructivo, de revisión y lleno de novedades. Es decir, un material que los médicos lean. Pero esto sucede lentamente, porque los modelos de negocio actuales funcionan en contra del cambio". Según este autor, las fuerzas opuestas a este 
cambio han motivado una especie de rebelión, favoreciendo la aparición del modelo de "acceso libre" que aspira a que toda investigación, la mayoría financiada con dinero público, se ofrezca gratuitamente y a texto completo en la red. Para Smith, la aparición en 1994 de la edición electrónica gratuita del B Med J (bmj.com), financiada sobre la base de la publicidad, ha sido el mejor florecimiento de la revista hasta ahora, pero también nos dice que las revistas se encuentran todavía en lo que sería su fase equivalente a los primeros tiempos de las películas; el cine sonoro todavía no ha aparecido. No obstante, diversas instituciones como BioMed Central, la Public Library of Science, US National Institutes of Health, el Howard Hughes Medial Institute, o el Wellcome Trust, entre otras, han emprendido esfuerzos financieros y logísticos para "catalizar la revolución pendiente en las publicaciones en ciencias biomédicas, proporcionando una demostración del valor y practicabilidad de la publicación de acceso libre".

Con el sistema vigente o convencional, la investigación médica, financiada fundamentalmente por los gobiernos, las universidades y fundaciones sin ánimo de lucro, está disponible tan sólo para una pequeña fracción de los potenciales lectores porque aparecen en revistas que pocas instituciones o particulares pueden pagar, y cuando lo hacen es con costes cada vez más altos; así los pagadores de impuestos, que ya han hecho su aportación económica para que se realice la investigación, deben pagar por ella otra vez ("taxpayers have already paid for this research-why should they pay for it again?" $)^{2,4,5,20,22,23}$. Este doble pago convierte la publicación médica en un lucrativo negocio que comporta unos beneficios de 7000 millones de dólares por año a las empresas editoriales. Como consecuencia, el Wellcome Trust del Reino Unido concluye en un análisis que "la publicación de los resultados de la investigación cientifica no se traduce en el interés de los científicos y del público, sino que es por el contrario dominada por el intento comercial o del mercado en mejorar sus propias posiciones". Se ha calculado que por las tasas de suscripción y circulación de las publicaciones, la comunidad científica está pagando actualmente alrededor de 4500 dólares por artículo, cuando tan sólo por una tercera parte (1500 dólares) se podría hacer llegar el mismo a la totalidad de sus miembros, en vez de al cada vez menos nutrido grupo de suscriptores; ésta es la cantidad que la Public Library of Science carga por articulo aceptado para cubrir los costes de su procesamiento ("peer review \& technical editing") y su posterior distribución electrónica haciéndolo así disponible sin cargos en su propia fuente "website", en el PubMed Central, y en el archivo digital libre de la National Library of Medicine. Este modelo comúnmente etiquetado como "authors pays", fue iniciado por BioMed Central que publica ya más de 100 "online journals" y supone un cambio de paradigma en relación con el modelo convencional "reader pays" de las suscripciones a las revistas. Así, muchas instituciones que constatan la diferencia en costes de la distribución de un articulo a través de la web (coste próximo a cero), con los de una separata de un articulo que conlleva los cargos de papel, impresión, ensamblado y correo de superficie, han emprendido una guerra feroz contra las firmas editoriales, (por ejemplo Elsevier) que está conduciendo en muchos casos, tras un tedioso "regateo", a la ruptura de la relación comercial cancelando el envío de originales a tales editoriales.

Si bien la idea del "acceso libre" a las publicaciones biomédicas es un objetivo a perseguir, la cuestión crítica es cómo hacerlo viable en términos económicos y si finalmente podrá generalizarse. Sostener el proceso de revisión por pares para depurar miles de manuscritos de los que sólo una pequeña parte van a ser finalmente editados, es un proceso muy caro al que se debe añadir después el coste repercutido por los editores de revistas en papel; ¿Bastarán los 1500 dólares por artículo para cubrir los costes de producción de una revista de alta calidad en la que más del $90 \%$ de los manuscritos revisados son finalmente rechazados?

¿Qué fuerzas o instituciones pueden limitar este idílico proyecto globalizador? Fundamentalmente los editores médicos cuyos negocios son los más rentables de la industria de los $\operatorname{media}^{4,5}$, pero también las propias instituciones académicas actúan como freno. En efecto, éstas se apoyan fuertemente en la consideración del factor de impacto como criterio para valorar la calidad de la investigación publicada, y el atractivo para los investigadores de las revistas con alto impacto disminuiría automáticamente al tener que cambiar el modelo de publicación habitual por otro nuevo que puede conllevar una bajada del mismo. Así pues, la implantación del modelo de "acceso libre", sea cualesquiera quien pague por la publicación (autor o la institución que le emplea) no resulta fácil y una alternativa, puesta ya en marcha por algunas publicaciones (i.e. en el área de la entomología), es ofertar al autor la opción de pagar los costes del procesamiento del articulo (modelo "autor pays "), que estaría así disponible para todo el mundo de manera gratuita y sería mejor conocida, o no pagar (modelo "reader pays "), lo que limitaría la disponibilidad del mismo a tan sólo los suscriptores. En cualquier caso, y en tanto se produce una evolución adicional, la mayor parte de la investigación biomédica original que se produce en el mundo permanece oculta a la mayor parte de los lectores que podrían usarla, por lo que la implantación del sistema de "acceso libre", que se considera imparable, se ha comparado acertadamente a la apertura de la cueva de Sésamo en publicación científica ${ }^{4}$.

Para empeorar las cosas, y a causa de los mecanismos fraudulentos que hacen posible en la práctica el acceso libre a muchas publicaciones que se esfuerzan inútilmente en 
protegerse del mismo, se ha desencadenado recientemente una fuerte reacción por parte de muchos cuerpos editoriales y revistas para evitar el acceso "pirata". Paradójicamente la propia revista British Medical Journal, pionera como dijimos en la política del libre acceso, ha sido la primera en cerrar filas creando su propia editorial de revistas y libros, y exigiendo naturalmente el pago por la disponibilidad de los mismos. Esta tendencia avanza de manera explosiva y cada vez más revistas aisladas o grupos de revistas están haciendo lo mismo; la única ventaja que presenta este cambio es rebajar los costes editoriales en comparación con la situación anterior, cuando editoriales independientes cargaban la publicación con costes disparatados. Una tendencia o alternativa esperanzadora es crear organismos nacionales que afronten de una vez los costes para hacer después libre el acceso a todos los miembros de la comunidad científica nacional; este es el caso de la National Electronic Library for Health del Servicio Nacional de Salud del Reino Unido. En nuestro país existen organismos diversos (Facultades, la Agencia Lain Entralgo, etc) que se aproximan a este modelo, pero no alcanzan la universalidad, multiplicándose así de manera inútil los costes por espacio físico, personal y tecnología.

Neurocirugía está disponible libremente en la red y la SENEC asume el coste de su libre difusión. Por tanto, nuestra revista "ya cumple" con el deseable proyecto de la edición universal de libre acceso, por lo que tiene poco que hacer en este sentido. Quizás el único punto a considerar en un futuro sería la aplicación del modelo ELPS, (electronic long - paper short = es decir utilizar simultáneamente una edición "electrónicamente larga" y otra "corta en el papel) que se utiliza ya en algunas publicaciones con una reducción considerable de los costes de la edición en papel al quedar ésta abreviada ${ }^{22}$. Algunas publicaciones en nuestro país, como la Revista Española de Cardiología, editan dos versiones extensas en inglés y en castellano en la red y una versión más corta en nuestra lengua en papel.

En relación con la revisión por pares, Smith opina que este proceso se encuentra aún en la era oscura ${ }^{22}$; en efecto, existe evidencia científica que "el sagrado proceso de la revisión por pares es lento, caro, ineficaz, una lotería, parcial, incapaz de detectar el fraude y propenso al abuso y las pruebas hacia el lado de su valoración positiva son escasas; sin embargo, y a causa de nuestra falta de imaginación, sigue siendo el sistema menos malo, por lo que deberíamos de mejorarlo en vez de suprimirlo. Mi visión es que una caja negra tosca debería transformarse en una disertación científica abierta mantenida a la vista y en tiempo real en la red, si bien esta visión futurista no es ampliamente compartida en el BMJ...". Según este autor, tampoco el uso de formatos estructurados para la presentación de estudios, como por ejemplo el CONSORT, ha logrado un desarrollo importante y la mayoría de las publi- caciones no exigen aún a los autores que los utilicen ${ }^{17,19}$.

Tras la celebración del $4^{\circ}$ Congreso Internacional en el año 2003 sobre el proceso de la "revisión por pares", D. Rennie, el editor de JAMA, extracta el siguiente párrafo de una editorial denunciando los decepcionantes estándares que prevalecen en el teóricamente riguroso proceso de selección de los artículos científicos que finalmente aparecen en las revistas ${ }^{20}$ : "One trouble is that despite this system (el "peer review') anyone who reads journals widely and critically is forced to realize that there are scarcely any bars to eventual publication. There seems to be no study too fragmented, no hypothesis too trivial, no literature citation too biased or too egotistical, no design too warped, no methodology too bungled, no presentation of results too inaccurate, too obscure and too contradictory, no analysis too self-serving, no argument too circular, no conclusion too trifling or too unjustified, and no grammar or syntax too offensive for a paper to end up in print". A continuación nos dice Rennie que si bien los esfuerzos para sistematizar y mejorar las revisiones de los artículos enviados a una revista y la manera de reportar los ensayos clínicos o los metaanálisis han llevado a un considerable progreso, el lector medio que viaje por el campo de la literatura médica con espíritu crítico, encontrará todavía con frecuencia los defectos apuntados. En efecto, más recientemente, este autor hace el siguiente comentario "las revistas médicas siguen plagadas de errores metodológicos, que mejoran poco y lentamente a pesar de los intentos de corrección de este extendido problema; por otra parte los editores apenas hacen algo por subsanarlos". D. Altman, experto asesor en estadística del BMJ, advirtió que a causa de dichos errores muchos estudios publicados alcanzan conclusiones falsas, y añade que, a pesar de ser éste un hecho bien conocido, el progreso en su control sigue siendo muy lento, y advierte que todavía muy a menudo la investigación médica se hace por personal no entrenado, que persigue objetivos equivocados, como por ejemplo el progreso en sus carreras ("engordar el currículo") ${ }^{23}$.

A pesar del tabú de que los autores no deben conocer en principio la identidad de los revisores, Rennie ha defendido la necesidad de adoptar "open peer review systems, whereby authors and eventually readers know the identity of reviewers ${ }^{\prime 21}$. Para este conocido editor "los argumentos supuestamente éticos en contra del proceso abierto de revisión por pares son lamentables e inconsistentes". Godlee ${ }^{9}$ también censura el dogma de que es mejor ocultar la identidad de los revisores, alegando que el sistema abierto tiene ventajas éticas, carece de inconvenientes colaterales importantes, es perfectamente practicable y mejora el crédito y la imagen científica de los propios revisores. Las experiencias de algunas revistas como el British Medical Journal o de BioMed Central han demostrado las ventajas del sistema abierto de revisión, y también el International Committee 
of Medical Journals Editor apoya la iniciativa de hacer más transparente este proceso, permitiendo que autores y revisores se comuniquen directamente a través de la red conociendo sus identidades. Algunas revistas se mueven incluso hacia un sistema de "pre-publication review" a cargo de los lectores, cuyas acertadas indicaciones podrían ser finalmente adoptadas por los autores para revisar su trabajos 9 .

Otro aspecto que tocan $\mathrm{Smith}^{22}$, y otros editorialistas como Rennie y cols ${ }^{20,21}$ es el de la autoría de los trabajos; es bien sabido que muchos manuscritos incluyen firmantes que no han hecho nada o bien poco, excluyendo por contra a otras personas que han realizado muchísimo esfuerzo. Por ello se preconiza moverse hacia un sistema que cambie la "authorship" por la "contributorship", en el que quienes contribuyen al estudio describan exactamente que es lo que hicieron.

Teniendo en cuenta los anteriores comentarios y otros ${ }^{13}$ de reconocidas autoridades en el campo de la publicación biomédica, está claro que la obligación de cualquier comité editorial es evitar los vicios y desviaciones inherentes al sistema de revisión por pares y tratar siempre de mejorar la pobre percepción que la mayoría de los autores tienen acerca del mismo ${ }^{25}$, pero también es incuestionable que los autores deben preparar mejor sus manuscritos persiguiendo siempre el rigor metodológico; sin buenos manuscritos ningún cuerpo editorial puede hacer un trabajo fructífero y digno, y ninguna revista puede perdurar. En cualquier caso, el empeño de Editor y revisores de Neurocirugía debería ser aproximarse al máximo a los nuevos estándares en el proceso de revisión, y el de los autores aplicarse una disciplina metodológica más estricta, recurriendo a cualquiera de las múltiples guías y recomendaciones metodológicas de libros y artículos enfocados a la escritura y estructuración de un artículo científico ${ }^{1,6,17}$. Cómo dinamizar y convertir Neurocirugía en un foro abierto, es una difícil pregunta que hemos de empeñarnos en contestar.

\section{Publicar en Neurocirugía. ¿Tiene razón de existir una revista como la nuestra?}

Las publicaciones neuroquirúrgicas representan tan sólo el $0.3 \%$ del total de lo editado en las revistas médicas incluidas en los bancos mayores de la literatura biomédica. Si bien este dato revela la limitada importancia de la contribución de los neurocirujanos al conocimiento global de la ciencia médica, también enmascara la relevancia de la actividad científica desarrollada por la comunidad neuroquirúrgica internacional, y en ningún caso debe desanimarnos a comunicar aquello que consideremos digno de ser compartido. Si aceptamos esto, surge la cuestión crítica de porqué, para qué y qué publicar en Neurocirugía.

Alguien dijo que lo que no se escribe se lo lleva el viento, y es nuestra opinión que a lo largo de la vida pro- fesional todos observamos algo digno de ser referido. Sin embargo, una gran mayoría de neurocirujanos no dejan ninguna huella escrita de su trabajo, escudándose en el argumento de que todas sus observaciones son comunes o repetitivas, y que el escaso valor añadido que aportarían a sus colegas no compensa el esfuerzo y tiempo necesarios para elaborar un escrito presentable. Aunque es cierto que sólo unos pocos producen de manera nítida conocimiento nuevo, (alguien reduce este grupo a tres o cuatro genios por siglo, pongamos el ejemplo de Pasteur), no lo es menos que el conocimiento progresa porque la acumulación de constataciones menores crea el marco o sustrato para que algunas mentes privilegiadas generen el salto más allá del límite que siempre separa lo viejo o ya sabido, de lo nuevo. Se ha dicho que progresamos porque nos subimos a hombros de los gigantes que nos preceden, pero los genios apoyan sus pies en las minúsculas aportaciones de otros muchos.

Nuestro tiempo se caracteriza por la facilidad de la comunicación, que asegura la inmediata circulación de cualquier aportación por mínima y remota que sea, pero al mismo tiempo la sobreabundancia de lo comunicado dificulta cada vez más la orientación del lector que pretende discernir lo nuevo. Este reto de la comunicación obliga a replantear constantemente cual debe ser nuestra actitud como profesionales inquietos por conocer, y algunos se preguntan si no es negativo o improcedente sobrecargar el ya abotargado depósito de publicaciones con contribuciones menores, propiciando así lo que se ha venido en llamar "polución científica" que conduce a la "fatiga informativa" del destinatario, que a veces acaba convirtiéndose en lo que Umberto Eco ha llamado un "imbécil superinformado". Por eso, a uno se le ocurre que quizás sería bueno llevar a cabo una reacreditación de las revistas incluidas en las principales redes de datos, aplicando niveles de exigencia progresivamente crecientes, hasta dejar un número relativamente pequeño en cada especialidad médica permitiendo así elevar a su vez el umbral de exigencia de calidad (sobre todo originalidad) de los artículos publicados.

Las razones para la creación y mantenimiento de una nueva revista científica que representa un ínfimo canal en la caudalosa corriente del conocimiento son varias. Neurocirugía es el órgano de expresión de la SENEC, y tan sólo el cometido de circular la información relacionada con la planificación y control del ejercicio profesional de sus miembros en las vertientes asistencial, científica y docente, tanto a nivel del pregrado como del postgrado, justificaría su existencia ${ }^{3,7,14-16,18}$. Esto es así siempre que la Sociedad Científica sea vital y creadora, lo que a su vez depende de la fuerza innovadora y el nivel profesional, tanto académico como científico, de sus miembros, y que además en el caso particular de España la vitalidad no se vea debilitada por la fragmentación de lo que debería ser un esfuerzo colectivo que nos permita tener una mayor presencia y perdurar en el 
competitivo escenario internacional.

Pero las revistas nacionales no deben ocuparse tan solo de asuntos parroquiales dejando la publicación de la producción científica propia para un número reducido de revistas en cada rama de la medicina. Aunque es loable tratar de "colocar" algunos de nuestros trabajos en revistas con un alto factor de impacto, Neurocirugía es un foro receptivo y de fácil acceso para reflejar la actividad científico-académica de los neurocirujanos españoles, y esta ventaja debería resultar particularmente atractiva para los más jóvenes que pueden batir en ella sus primeros cobres. ¡Cuanto hubiera apreciado Cajal disponer de una revista como Neurocirugía, que siendo española tuviera una difusión universal en los principios de su carrera investigadora, cuando lamentaba el aislamiento casi absoluto de su genial producción científicaj. Escribir trabajos científicos de cualquier alcance supone reflexionar, y toda reflexión lleva en sí misma el germen de la búsqueda de lo inexplicado, que una vez entendido ha de compartirse con los pares. En este sentido resulta ilustrativa la descripción del nacimiento de una de la primeras sociedades científicas, la Real Sociedad de Londres, en el siglo XVII que se puede leer en una biografía de Isaac Newton aparecida recientemente ${ }^{8}$. pp: 75-76 .. "La Real Sociedad había nacido para estimular el flujo de información, para enaltecer la comunicación y condenar el secretismo... la ciencia no existía aún, pero sus miembros fundadores la entendían como una empresa estatal, e imaginaban una red global, un imperio a favor del conocimiento. Aquellos que se afanaban en captar en su totalidad el tejido de la naturaleza deberian tener los ojos en todas partes y recibir información de todos los rincones de la tierra, deberían poseer un conocimiento universal constante; todos los descubrimientos deberían llegar a su conocimiento, y los tesoros de todos los tiempos pasados deberían abrirse ante ellos... Y en qué idioma? Entre las funciones de la Real Sociedad se incluía la traducción de textos, obligados como estaban a enfrentarse a los múltiples idiomas autóctonos de Europa, o incluso a lenguas tan desconocidas como las que se decía que hablaban en países como India o Japón. El latín servía para la uniformidad, pero a los fundadores de la Real Sociedad les preocupaba de manera explícita la utilización de cualquier idioma... " ¿Qué visión tan profética y fecunda la de Newton, Barrow y demás cofundadoresi . ¿Acaso no se vislumbra ya en sus aspiraciones y propósitos la web de nuestro siglo, y no plantearon acaso en toda su problemática la cuestión del uso de diferentes lenguas en la comunicación científica?

En relación con el asunto de la lengua a emplear en nuestras aportaciones a la literatura ${ }^{10,24}$, se puede decir que nuestros colegas italianos acertaron al elegir el inglés para publicar los trabajos remitidos al Journal of Neurosurgical Sciences dado el escaso número de italiano parlantes en el mundo. Por otro lado, resulta admirable el celo de nues-
2006; 17: 89-97

tros colegas franceses en defender y preservar su lengua, si bien hay que reconocer que los mejores artículos de la neurocirugía francesa ya no se publican en Neurochirurgie, sino en las revistas norteamericanas. En nuestro caso el asunto es más espinoso y discutible, ya que por un lado el castellano es hablado por más de 300 millones de personas, y aún cuando el eco de nuestra revista en Centro y Sudamérica sea todavía limitado, no cabe duda que tiene sentido permitir la elección entre el castellano y el inglés como lenguas para publicar artículos en Neurocirugía. Quizás sería deseable que la proporción de artículos publicados en inglés se incrementara, ya que es más probable que un artículo sea leído y citado si está escrito en esta lengua, que si tan sólo se oferta su "abstract" acompañando a su versión en castellano; el texto completo en inglés aumenta las expectativas del autor de ver citado su trabajo, e incrementa de paso el factor de impacto de Neurocirugía, siendo este el motivo por el que nuestro grupo ha publicado en ella algunos artículos escritos en ingles que no fueron enviados previamente para consideración a otras publicaciones. No obstante, se puede comprobar cómo artículos publicados en castellano son citados sobre las base del "abstract" en revistas de habla inglesa. En cualquier caso, parece apropiado dejar a la elección de los autores esta decisión, ya que en definitiva representa una facilidad adicional. El castellano es imprescindible para publicar todo tipo de contribuciones relacionadas con la práctica y la vida neuroquirúrgica en nuestro ámbito y además su exclusión limitaría la llegada a muchos lectores y disminuiría la oferta de artículos escritos por autores castellano parlantes. Algunos estudios indican que si bien la lectura en la pantalla de un ordenador o en papel no marcan ninguna diferencia en la capacidad para retener la información ofertada, la lectura de un artículo en la lengua materna proporciona una retención de datos mayor que cuando lo leído esta escrito en otra lengua ${ }^{11}$.

\section{EI hábito y la utilidad de la escritura}

Escribir es un trabajo duro, sobre todo en los principios, pero se dice que la función hace al órgano. Los ingleses tienen un dicho que reza "Avoid sitting thinking about writing, write;". Escribir enseña no sólo a hablar mejor, sino a discurrir y reflexionar, y hace posible la producción de la pequeña obra propia. Sólo si nuestros colegas jóvenes escriben mucho y bien, la neurocirugía española en general, y la revista Neurocirugía en particular, tendrán alguna resonancia en el extenso ámbito de las neurociencias. En su libro "Los tónicos de la voluntad", cuya lectura en algún momento de la residencia enriquecería siempre al neurocirujano joven, Cajal dice que uno de los objetivos de nuestro trabajo bien hecho es "aumentar el caudal de ideas españolas circulando por el mundo, granjeando respeto $y$ simpatía para nuestra ciencia". Pero aparte del carácter 
instrumental de la escritura para difundir una contribución científica más o menos relevante, existe evidencia científica clara de que la escritura expresiva, exploradora y reflexiva alivia la tensión psíquica y potencia el entendimiento, mejorando así nuestro rendimiento intelectual; se ha demostrado también que escribir o narrar las propias experiencias, entre ellas algunas vividas con los pacientes, es muy importante para entrenar a los médicos, y la emergencia y exuberante florecimiento de la llamada "narrative based medicine" lo ilustran bien ${ }^{25}$. Alguien dijo que no somos más que nuestras historias, y escribir y reescribir narraciones nos mantiene alerta, inquisitivos, vivos y flexibles. Escribir permite al autor expresar y clarificar experiencias, pensamientos e ideas que son problemáticas y turbadoras o difíciles de delimitar, y tiene una función formativa más profunda que hablar o pensar. Una ventaja de lo escrito frente a lo hablado o lo pensado, es que permanece sin variación en la página que lo vio nacer, pudiendo así ser trabajado al día siguiente o un año después si se quiere, o por el contrario acabar siendo destruido sin tan siquiera ser releído por el autor.

Para enfatizar la importancia del gusto por la escritura y su utilidad para la expresión científica, nada mejor que otro breve retazo de la biografía de Newton ${ }^{8}$, en la que se aprecia cómo el pensamiento de quien desveló con certeza nuestro lugar en el cosmos, se forjó en gran manera sobre la disciplina de la escritura.

"A los dieciséis años, como estudiante becario en el Trinity College de Cambridge sumergía el cañón de su pluma en tinta de agalla de roble, y escribía en latín, con letra minúscula, comprimiendo las palabras una junto a la otra... A estas alturas había escrito más de un millón de palabras y apenas había publicado nada. La primera fábrica de papel de Inglaterra se acababa de abrir cuando el nació; el papel era muy caro, y la palabra escrita influía muy poco en la vida cotidiana... Gran parte de lo que la gente pensaba seguía sin plasmarse por escrito y la mayor parte de lo que escribía permanecía oculto o se perdía. Newton escribía para sí, indiferente al hambre o al sueño. Escribía para calcular y anotaba números en hileras de trazos alargados y anchas columnas. Hacía cálculos de la misma manera que la gente suele soñar despierta. El flujo de su pensamiento iba y venía entre el inglés y el latín. Escribia para leer, copiando libros y manuscritos palabra por palabra, a veces el mismo texto una y otra vez. Con mayor determinación que placer, escribía para razonar, para meditar y para ocupar su mente tan febril. La paciencia de Newton no tenía límites. La verdad, diría mucho después era "hija del silencio y de la meditación". Asi desarrolló una habilidad ilimitada para generalizar, para trasladarse de unos cuantos casos particulares conocidos, al universo de todos los casos; resultaba que la mente humana, a pesar de hallarse confinada dentro de un estuche, es capaz de discernir el infinito y de medirlo.... En aquel entonces la información circulaba débilmente y de forma perecedera entre los humanos pero Newton creó en la más estricta soledad un método que abrió la puerta a un nuevo universo asentado en el espacio y el tiempo, un sistema del mundo ".

La poderosa mente de Newton necesitó crear las palabras aún no alumbradas para acuñar términos, conceptos y realidades físicas todavía vagamente definidos (unidades, cantidades, movimientos, fuerzas, etc) y poder así comunicar sus reflexiones y hallazgos concretando de esta manera su obra científica. A los veinticuatro años estaba convencido de que podría ordenar toda la ciencia del movimiento con sólo encontrar el léxico apropiado, con sólo ser capaz de poner las palabras en el orden correcto. Nosotros no poseemos la poderosa mente de Newton, pero también tenemos planteados enigmas que resolver, y para incrementar nuestro entusiasmo podremos siempre inspirarnos en su titánica determinación y método de trabajo.

\section{Bibliografía}

1. Browner, W.S. Publishing and Presenting Clinical Research. Baltimore, Lippincot Williams \&Wilkins, 1999.

2. Butler, D.: Who will pay for open access? Nature 2003; 425: 554-555.

3. Carrillo, R.: Censo de neurocirujanos en España. Neurocirugía 1997; 8: 25-28.

4 Delamonthe, T., Godlee, F., Smith, R.: Scientific literature,s open sesame? BMJ 2003; 326: 945-946.

5. Delamonthe, T., Smith, R.: Open access publishing takes off. The dream is now achievable. BMJ 2004; 328: 1-3.

6. Eastwood, D., Derish, P.A., Berger, M.S.: Biomedical publication for neurosurgery residents: A program and guide. Neurosurgery 2000; 47: 739-749.

7. Esparza, J., Carrillo, R.: Estudio sobre el sistema de guardias en la Sociedad Española de Neurocirugía. Neurocirugía 2003; 14: 81-106.

8. Gleick, J.: Isaac Newton. RBA Libros S.A, Barcelona, 2005

9. Godlee, F.: Making reviewers visible. Openness, accountability and credit. JAMA 2002; 287: 2762-2764.

10. Greenhalgh, T., Hurwitz, B.: Narrative based medicine: dialogue and discourse in clinical practice. London: BMJ Publications, 1998.

11. Gulbrandsen, P., Schroeder, T.V., Mirelae, J., Nylenna, M.: Paper or screen. Another tongue or English. Which is better? JAMA 2002; 237: 2851-2855.

12. Ibañez, J., Sahuquillo, J., Poca, M.A., Arikan, F.: La incorporación de Neurocirugía al Journal of Citation Reports: Análisis bibliométrico de la producción científica neuroquirurgica española. Neurocirugía 2000; 11: 329-350.

13. Jefferson, T., Alderson, P., Wagner, E., Davidoff, F.: 
Effects of editorial peer review: a systematic review. JAMA 2002; 287: 2784-2786.

14. Lobato, R.D., Poza, M., Lagares, A.: Recomendaciones sobre el horario del residente y su entorno formativo. Neurocirugía 2002; 13: 146-154.

15. Lobato, R.D., Alen, J.F., Alday, R., Gómez, P.A., Lagares, A.: El Nuevo Programa de Residencia en Neurocirugía en España. Neurocirugía 2003; 14: 281-294.

16. Lobato, R.D.: La formación investigadora en los programas de residencia en Neurocirugía. Análisis de la situación en otros países desarrollados y propuestas para complementar el actual programa en España. Neurocirugía 2002; 13: 146154

17. Moher, D., Jones, A., Lepage, L.: Use of the CONSORT statement and quality of reports of randomized trials: a comparative before-and-after evaluation. JAMA 2001; 285 : 1992-1995.

18. Poza, M.: Formacion de residentes en neurocirugia. Neurocirugía 2001; 12: 388-397.

19. Rennie, D.: CONSORT revised-improving the reporting of randomized trials. JAMA 2001; 285: 2006-2007.

20. Rennie, D.: Fourth international congress on peer review in biomedical publication. JAMA 2002; 21: 27592760 .

21. Rennie, D., Yank, V., Emanuel, L.: When autorship
2006; 17: 89-97

fails: a proposal to make contributors accountable. JAMA 1997; 278: 579-585.

22. Smith, R.: Travelling but never arriving: reflections of a retiring editor. Twenty five years of adventure, discovery and conservatism BMJ 2004; 329: 242-244.

23. Smith, R.: Medical editor lambasts journals and editors. BMJ 2001; 323: 651.

24. Trojanowski, T., Rabow, I., Rabow, L.: Neurosurgical publications in European Journals. Acta Neurochir (Wien) 1992: 116: 98-106.

25. Weber, E.J., Katz, P.P., Waerckerle, J.F., Callahan, M.L.: Autor perception of peer review. Impact of review quality and acceptance on satisfaction. JAMA 2002; 287:27902793.

Agradecimientos: Queremos expresar nuestro reconocimiento a Javier Ibañez por su ayuda en la revisión de este manuscrito

Lobato, R.D.; González,P.; Gómez, P.A.; Alday, R.; Lagares, A.; Alen, J.F.: El cambio en el proceso editorial de la revista Neurocirugía. Neurocirugía 2006; 17: 89-97.

Correspondencia postal: R. D. Lobato. Servicio de Neurocirugía. Hospital "12 de Octubre", Avda de Córdoba /sn. 28041 Madrid. 